\title{
An update on coeliac disease from the NHS England National Centre for Refractory Coeliac Disease
}

\author{
Authors: Anupam Rej ${ }^{A}$ and David S Sanders ${ }^{B}$
}

Coeliac disease (CD) is a common autoimmune-mediated gluten sensitive enteropathy, with a prevalence of around $1 \%$. While the incidence of CD has increased over the last 2 decades, many cases still remain undiagnosed. The presentation of $C D$ is variable and can be subtle, with it being important to explore both gastrointestinal and extraintestinal features. The cornerstone of management is adherence to a strict gluten free diet, which requires support and education from an expert gastrointestinal dietitian. Persisting symptoms in individuals requires re-evaluation, with repeat duodenal biopsies sometimes required. Refractory $C D$ affects a small subset of individuals with $C D$, requiring specialist input.

\section{Introduction}

Coeliac disease (CD) is a common autoimmune mediated gluten sensitive enteropathy, with a reported pooled prevalence of $0.7 \%$ based on biopsy confirmation, and $1.4 \%$ based on serology. ${ }^{1}$ While initially thought to be a diagnosis predominantly of children in a European population, it is now known that CD can affect any age, commonly diagnosed between the 4 th and 6 th decade (mean age of 45 years at diagnosis), with a higher incidence in females. ${ }^{2,3} \mathrm{CD}$ is a global disease, increasing in incidence over the last 2 decades. 1

\section{Clinical presentation}

Despite an increase in incidence of $C D$, many remain undiagnosed. ${ }^{3}$ Diagnosis can be delayed, and symptoms may be present for a mean of 11 years before diagnosis. ${ }^{4}$ This delay in diagnosis is associated with a prolonged and significant decrease in quality of life.

Historically, it was thought that CD solely presented with signs and symptoms of malabsorption (such as diarrhoea, weight loss and steatorrhoea). ${ }^{5}$ While individuals may present like this, known as classical CD, it is now known that individuals can present in a multitude of ways. ${ }^{5}$ Individuals may present without symptoms and signs of malabsorption, known as non-classical CD. ${ }^{5}$ These can be gastrointestinal (GI; such as constipation and abdominal pain) but can also can be extra-intestinal (such as osteoporosis,

Authors: ${ }^{\text {A }}$ gastroenterology clinical fellow, Royal Hallamshire Hospital, Sheffield, UK; B honorary professor of gastroenterology and consultant gastroenterologist, Royal Hallamshire Hospital, Sheffield, UK dermatitis herpetiformis, neurological manifestations, infertility, deranged liver function tests and thyroid dysfunction). ${ }^{5}$

Presenting features may be subtle (such as fatigue, recurrent or severe mouth ulcers, or haematinic deficiencies). ${ }^{6}$ A history of autoimmune disease should be sought (such as type 1 diabetes and thyroid dysfunction), as well as a family history of $C D$, with the prevalence being around eight times higher in first degree relatives. ${ }^{3,6}$ In addition, individuals presenting with irritable bowel syndrome (IBS) should be tested for CD. ${ }^{6} \mathrm{CD}$ should also be considered in some chromosomal disorders such as Down's syndrome and Turner's syndrome.

\section{Diagnosis}

Individuals with suspected CD must be on a gluten containing diet prior to both serological and histological testing. They should be

\section{Key points}

Coeliac disease is common, with a prevalence of around $1 \%$; many patients are undiagnosed and can present with subtle symptoms to a wide variety of medical specialties.

Diagnosis can be made by positive serology (IgA-tTG/IgAEMA) in conjunction with confirmatory histology (Marsh $3 a$ or above), but during the COVID-19 pandemic, a serologybased diagnosis can be made in select individuals.

Management is with a lifelong strict gluten free diet, requiring the input of a specialist dietitian.

Persisting symptoms on a gluten free diet requires reevaluation, occurring in up to a third of individuals, with repeat duodenal biopsies being the only effective method to assess for ongoing villous atrophy.

Refractory coeliac disease affects a small subset of patients with coeliac disease and may require treatment with steroids and immunomodulators.

KEYWORDS: coeliac disease, refractory coeliac disease, gluten, wheat

DOI: 10.7861/clinmed.2021-0025 
advised to consume at least $10 \mathrm{~g}$ of gluten daily (around four slices of bread) for 6 weeks prior to testing. This may be challenging for those with significant ongoing symptoms with gluten; in this scenario a shorter gluten challenge for 2 weeks may be considered, although the detection of villous atrophy is variable, reported between $26 \%$ and $68 \%$ using this approach. ${ }^{8,9}$

For serological testing, immunoglobulin A ( IgA) tissue transglutaminase (tTG) testing should be performed in the first instance. ${ }^{6}$ IgA-tTG has a high sensitivity and specificity for the diagnosis of $C D$, reported as mean of $94 \%$ and $97 \%$, respectively. ${ }^{10}$ Alternatively, IgA endomysial antibody (EMA) testing can also be used, and is recommended when IgA-tTG is weakly positive. ${ }^{6}$ While IgA-EMA can also be used in view of its high sensitivity and specificity (reported at $>86 \%$ and around $100 \%$, respectively), it has limitations, being a qualitative test open to inter-observer variability as well as being labour intensive to analyse. ${ }^{10}$ When assessing serology, IgA levels should be checked, owing to the fact of the high prevalence of IgA deficiency for individuals with $C D$, reported at $2.6 \%{ }^{3}$ In individuals with IgA deficiency, IgG-tTG serology should be performed. ${ }^{3}$ A small minority of individuals (3-5\%) have seronegative CD but positive histology. These individuals respond to a gluten free diet (GFD), and may present at an older age, with classical symptoms being more common. ${ }^{11}$ However, it is worth noting there are several other causes for seronegative villous atrophy (such as due to nonsteroidal anti-inflammatory drugs, Helicobacter pylori, common variable immunodeficiency, tuberculosis and autoimmune enteropathy). ${ }^{3,11}$

If an individual has positive coeliac serology, a diagnosis of CD is confirmed histologically by taking duodenal biopsies from the duodenal bulb and distal duodenum, with four biopsies doubling the diagnostic rate. ${ }^{3}$ Histological features of $C D$ include an increase in intraepithelial lymphocytes, crypt hyperplasia and villous atrophy (Fig 1). ${ }^{12}$ While duodenal biopsies remain the cornerstone to confirm diagnosis in individuals with adult CD, the European Society for Paediatric Gastroenterology, Hepatology and Nutrition (ESPGHAN) guidelines suggest a diagnosis of CD can be made in selected children and adolescents with high tTG titres ( $>10 \times$ upper limit of normal (ULN)), compatible human leukocyte (HLA) typing and positive IgA-EMA serology, without the requirement for a duodenal biopsy. ${ }^{13}$ This approach is now being evaluated in the adult population, with a recent large multicentre study demonstrating that a high tTG titre ( $>10 \times$ ULN) had a strong positive predictive value for identifying CD, reported between $95.2 \%$ and $100 \% .{ }^{14}$ In light of the COVID-19 pandemic, current interim British Society of Gastroenterology guidelines suggest a no biopsy approach for adults under the age of 55 years with high tTG titres ( $>10 \times$ ULN) and positive IgA-EMA provided they have no alarm symptoms. ${ }^{15} \mathrm{~A}$ no biopsy approach in selected groups of adults maybe the future for diagnosis globally, but hurdles remain, including the challenge of tTG assay standardisation. $^{3}$

The main genetic factors predisposing to $C D$ lie in the HLA regions, with the HLA class II genes HLA-DQA1 and HLA-DQB1 being key. ${ }^{7} \mathrm{HLA}$ typing is not used routinely in the diagnosis of $C D$, but may have utility in its exclusion, such as in individuals selftreated on a GFD who are unwilling to undergo a gluten challenge to have serological testing. HLA typing has a high negative predictive value for CD of greater than $99 \%$ but up to $40 \%$ of the general population may have a positive result, limiting its general use for diagnosis. ${ }^{13}$

\section{Management and monitoring}

Once a diagnosis of CD is confirmed, physicians should strongly recommend a GFD and support the individual to understand the reasons for this. This involves the strict dietary exclusion of wheat, rye and barley, ideally under the guidance of a specialist dietitian. ${ }^{3}$
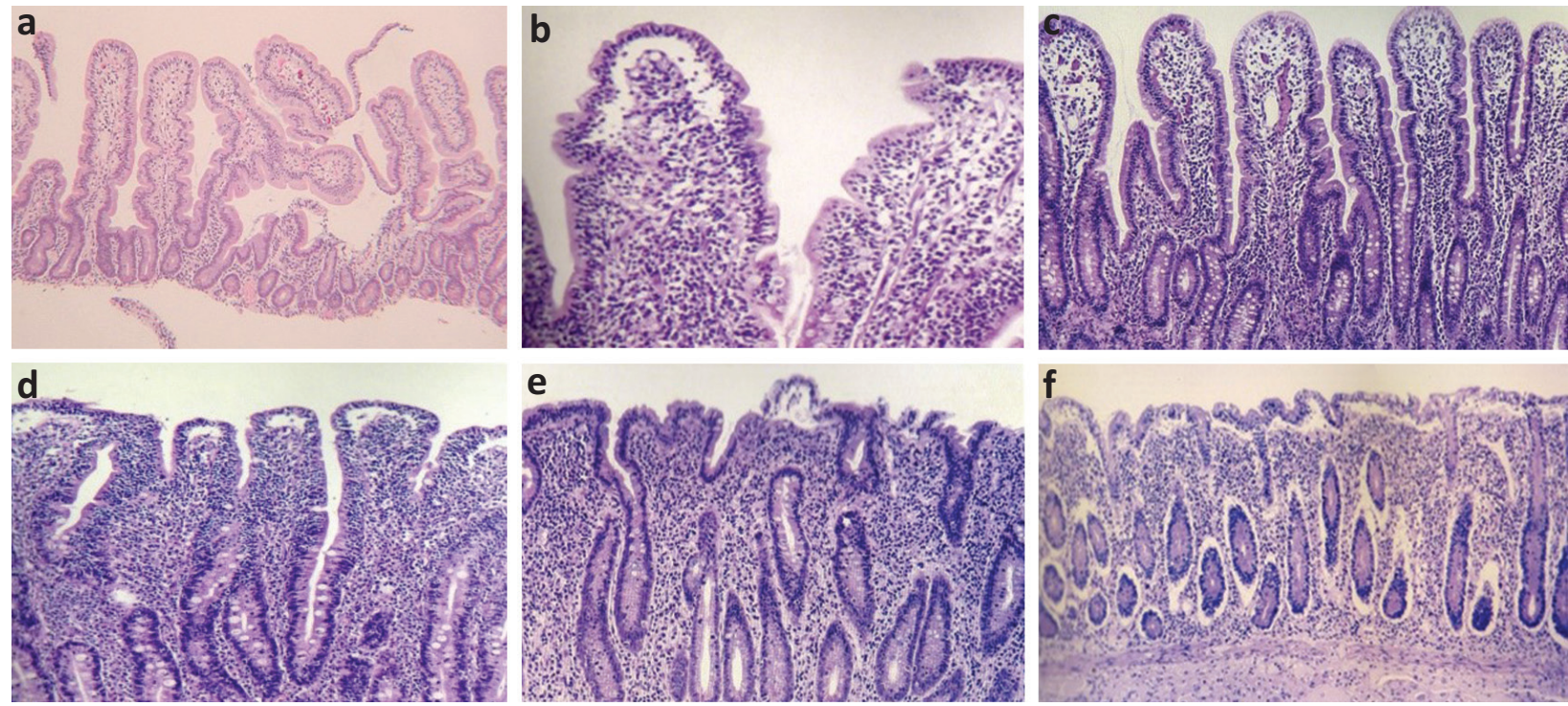

Fig 1. Modified Marsh criteria for classification of coeliac disease. ${ }^{17}$ Stage 3 (lower row) denotes histology consistent with diagnosis of coeliac disease. a) Stage 0: normal. b) Stage 1: increase in intraepithelial lymphocytes. c) Stage 2: increase in intraepithelial lymphocytes and crypt hyperplasia. d) Stage 3a: increase in intraepithelial lymphocytes, crypt hyperplasia and partial villous atrophy. e) Stage 3b: increase in intraepithelial lymphocytes, crypt hyperplasia and subtotal villous atrophy. f) Stage 3c: increase in intraepithelial lymphocytes, crypt hyperplasia and total villous atrophy. 


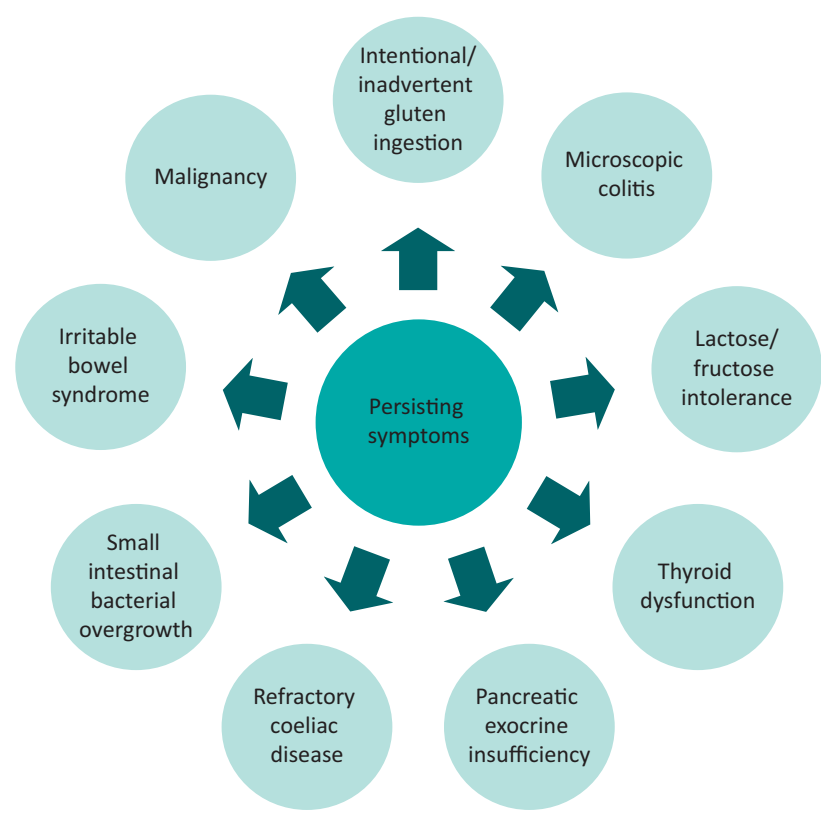

Fig 2. Diagnosis for persisting symptoms.

Individuals may be able to consume pure oats in small amounts, although gluten cross contamination is possible. ${ }^{3}$

Current National Institute for Health and Care Excellence (NICE) guidelines suggest annual follow-up for individuals with $C D$, which is commonly performed in primary care, although follow-up remains variable. ${ }^{6}$ Individuals appear to be keen to be followed up by a dietitian with a physician being available if required. At review, symptoms, osteoporotic risk, micronutrient deficiencies (iron, folate, B12 and vitamin D) and dietary adherence should be assessed., Some individuals with CD may have splenic hypofunction, with pneumococcal vaccination recommended for all. ${ }^{16}$

Up to a third of individuals with CD may have persisting symptoms despite being on a GFD, known as non-responsive CD (NRCD). ${ }^{17}$ The commonest cause is ongoing gluten exposure, which may be inadvertent or intentional. ${ }^{17}$ Adherence to a GFD can be challenging, with reported adherence in individuals with CD being between 42 and $91 \%{ }^{3}$ Currently, there are no effective non-invasive markers of GFD adherence, with point-of-care testing, dietary adherence questionnaires and serology having a poor sensitivity for the detection of villous atrophy. ${ }^{3}$ In view of this, repeat duodenal biopsies remains the gold standard for assessment. ${ }^{3}$ This assessment remains crucial in individuals with persisting symptoms, as mucosal healing is important to reduce the long-term complications in CD (such as osteoporosis and malignancy). ${ }^{3}$ Alternative diagnosis for persisting symptoms should also be considered (such as IBS, pancreatic exocrine insufficiency, microscopic colitis, lactose/fructose intolerance and thyroid dysfunction; Fig 2). ${ }^{17}$

A small minority of individuals with CD may have persisting villous atrophy despite strict adherence to a GFD. If the duration of GFD management has been greater than 12 months, and other diagnosis have been excluded, a diagnosis of refractory $C D(R C D)$ may be made. ${ }^{3}$ The prevalence of RCD is rare, reported in less than $4 \%$ of individuals with CD. ${ }^{3}$ Techniques such as flow cytometry on histological samples can be used to distinguish between type
1 RCD (RCD 1) and type 2 RCD (RCD 2), with RCD 2 having a poor prognosis of $50 \%$ at 5 years in comparison with $80-100 \%$ at 5 years in RCD 1. ${ }^{3}$ Ideally, these individuals should be managed at specialist centres, where therapies such as corticosteroids and immunosuppressants may be employed. ${ }^{3,17}$ It is worth noting that these therapies do not prevent the development of complications such as enteropathy-associated T-cell lymphoma, but may delay its onset. ${ }^{17}$ NHS England have selected Sheffield as the national centre for RCD (lead centre contact: david.sanders1@nhs.net), with Cambridge as the member centre.

\section{Future developments}

Currently, adherence to a GFD remains challenging. In view of this, portable gluten detection devices are now commercially available to assist individuals with inadvertent gluten exposure. ${ }^{18}$ However, with these devices being able to detect levels of gluten less than 20 parts per million, there are potential limitations with this approach of unknown clinical significance. ${ }^{18}$ As a result, this approach may lead to an excessive obsession with healthy eating (known as orthorexia nervosa), with further research required in this area to assess the utility of these devices. ${ }^{3}$

A promising area for the assessment of GFD adherence includes the use of gluten immunogenic peptides (GIPs), either via urine or faeces. ${ }^{3}$ It has been suggested that this approach may be a useful way to indirectly assess mucosal healing, although further research is required. ${ }^{19}$ In addition, GIPs are only transiently present for a few days post-gluten ingestion, which may limit its use if individuals alter their behaviour prior to testing. ${ }^{19}$

While the GFD remains the cornerstone for the management of $C D$, there has been a search for novel medical treatments for $\mathrm{CD}$, such as zonulin inhibitors and vaccination. ${ }^{20}$ However, most trials are currently within the pre-clinical phase, with few in phase II or III, and it is possible that medical treatments may play a useful role in the future as an adjunct to a GFD rather than replacement. $^{20}$

\section{Conclusion}

$\mathrm{CD}$ is a common disorder, presenting with both gastrointestinal and extra-intestinal manifestations, with diagnosis made by confirmatory serology and histology. Adherence to a GFD remains challenging, with repeat duodenal biopsy being key to assess individuals with persisting symptoms. While rare, RCD ideally requires management in a specialist centre to assess for complications.

\section{References}

1 Singh P, Arora A, Strand TA et al. Global Prevalence of Celiac Disease: Systematic Review and Meta-analysis. Clin Gastroenterol Hepatol 2018;16:823-36.

2 Sanders DS, Hurlstone DP, Stokes RO et al. Changing face of adult coeliac disease: experience of a single university hospital in South Yorkshire. Postgrad Med J 2002;78:31-3.

3 Rej A, Aziz I, Sanders DS. Coeliac disease and noncoeliac wheat or gluten sensitivity. J Intern Med 2020;288:537-49.

4 Green PHR, Stavropoulos SN, Panagi SG et al. Characteristics of adult celiac disease in the USA: results of a national survey. Am J Gastroenterol 2001;96:126-31.

5 Ludvigsson JF, Leffler DA, Bai JC et al. The Oslo definitions for coeliac disease and related terms. Gut 2013;62:43-52. 
6 National Institute for Health and Care Excellence. Coeliac disease: recognition, assessment and management: NICE guideline [NG20] NICE, 2015. www.nice.org.uk/guidance/ng20

7 Lebwohl B, Sanders DS, Green PHR. Coeliac disease. Lancet 2018;391:70-81.

8 Leffler D, Schuppan D, Pallav K et al. Kinetics of the histological, serological and symptomatic responses to gluten challenge in adults with coeliac disease. Gut 2013;62:996-1004.

9 Sarna VK, Skodje GI, Reims HM et al. HLA-DQ: gluten tetramer test in blood gives better detection of coeliac patients than biopsy after 14-day gluten challenge. Gut 2018;67:1606-13.

10 Penny HA, Raju SA, Sanders DS. Progress in the serology-based diagnosis and management of adult celiac disease. Expert Rev Gastroenterol Hepatol 2020;14:147-54.

11 Schiepatti A, Sanders DS, Biagi F. Seronegative coeliac disease: clearing the diagnostic dilemma. Curr Opin Gastroenterol 2018;34:154-8.

12 Oberhuber G, Granditsch G, Vogelsang H. The histopathology of coeliac disease: time for a standardized report scheme for pathologists. Eur ] Gastroenterol Hepatol 1999;11:1185-94.

13 Husby S, Koletzko S, Korponay-Szabo IR et al. European Society for Pediatric Gastroenterology, Hepatology, and Nutrition guidelines for the diagnosis of coeliac disease. J Pediatr Gastroenterol Nutr 2012:54:136-60.

14 Penny HA, Raju SA, Lau MS et al. Accuracy of a no-biopsy approach for the diagnosis of coeliac disease across different adult cohorts. Gut 2020 [Epub ahead of print]
15 Penny HA SD, Gillett H, Gillett P et al. BSG Interim Guidance: COVID-19 spcific non-biopsy protocol those with suspected coeliac disease. British Society of Gastroenterology, 2020. www.bsg.org.uk/ covid-19-advice/covid-19-specific-non-biopsy-protocol-guidancefor-those-with-suspected-coeliac-disease

16 Ludvigsson JF, Bai JC, Biagi F et al. Diagnosis and management of adult coeliac disease: guidelines from the British Society of Gastroenterology. Gut 2014;63:1210-28.

17 Penny HA, Baggus EMR, Rej A et al. Non-responsive coeliac disease: a comprehensive review from the NHS England National Centre for Refractory Coeliac Disease. Nutrients 2020;12;216.

18 Lerner BA, Phan Vo LT, Yates S et al. Detection of gluten in glutenfree labeled restaurant food: analysis of crowd-sourced data. Am J Gastroenterol 2019:114:792-7.

19 Moreno ML, Cebolla A, Munoz-Suano A et al. Detection of gluten immunogenic peptides in the urine of patients with coeliac disease reveals transgressions in the gluten-free diet and incomplete mucosal healing. Gut 2017;66:250-7.

20 Caio G, Ciccocioppo R, Zoli G et al. Therapeutic options for coeliac disease: What else beyond gluten-free diet? Dig Liver Dis 2020:52:130-7.

Address for correspondence: Dr Anupam Rej, NHS England National Centre for Refractory Coeliac Disease, Sheffield Teaching Hospital NHS Foundation Trust, Glossop Road, Broomhall, Sheffield S10 2JF, UK.

Email: anupam.rej@nhs.net 